\title{
On Ubiquitous Technology, a Digital World and their Influence on People's Feeling and Control of Presence in Everyday Life
}

\author{
Nađa Terzimehić \\ LMU Munich, Munich, Germany \\ nadja.terzimehic@ifi.lmu.de \\ Renate Häuslschmid \\ Faculty of Medicine and Medical Center \\ University of Freiburg, Freiburg, Germany \\ haeuslschmid@imbi.uni-freiburg.de
}

\author{
Ceenu George \\ LMU Munich, Munich, Germany \\ ceenu.george@ifi.lmu.de \\ Heinrich Hussmann \\ LMU Munich, Munich, Germany \\ hussmann@ifi.lmu.de
}

\begin{abstract}
Ubiquitous technology, e.g., smartphones or tablets, has created a continuously available digital world, drastically changing our feeling of being in the here and now - named presence. We thus increasingly shift between the real and the digital world, ranging from losing awareness of real surroundings to cutting out the digital world to truly be in the real one. In this work, we aim to explore the middle ground in between. We move beyond classic VR research on presence and look at everyday ubiquitous technology and its influence on presence in the real and digital world. By means of a focus group $(N=6)$ and a subsequent online survey $(N=36)$, we gathered individual notions of presence as well as experiences and situations in which people move between the worlds. We discuss the need to further explore presence and its dynamics across the real and digital worlds in everyday life.
\end{abstract}

ACM Reference Format:

Nađa Terzimehić, Ceenu George, Renate Häuslschmid, and Heinrich Hussmann. 2021. On Ubiquitous Technology, a Digital World and their Influence on People's Feeling and Control of Presence in Everyday Life. In CHI Conference on Human Factors in Computing Systems Extended Abstracts (CHI '21 Extended Abstracts), May 8-13, 2021, Yokohama, Japan. ACM, New York, NY, USA, 7 pages. https://doi.org/10.1145/3411763.3451831

\section{INTRODUCTION \& BACKGROUND}

"Whether we wear computers on our body, or have them embedded in our environment, the ability of computers to alter our perception of the physical world, to support constant connectivity to distant people and places, to provide information at our fingertips, and to continuously partner with us in our thoughts and actions offers much more than a new "killer app" - it offers the possibility of a killer existence." [1]

Permission to make digital or hard copies of all or part of this work for personal or classroom use is granted without fee provided that copies are not made or distributed for profit or commercial advantage and that copies bear this notice and the full citation on the first page. Copyrights for components of this work owned by others than the author(s) must be honored. Abstracting with credit is permitted. To copy otherwise, or republish, to post on servers or to redistribute to lists, requires prior specific permission and/or a fee. Request permissions from permissions@acm.org.

CHI '21 Extended Abstracts, May 8-13, 2021, Yokohama, Japan

(C) 2021 Copyright held by the owner/author(s). Publication rights licensed to ACM. ACM ISBN 978-1-4503-8095-9/21/05...\$15.00

https://doi.org/10.1145/3411763.3451831
In 2000, Abowd and Mynatt [1] envisioned that novel ubiquitous technology will lead to everyday human-computer interaction being more intertwined with and across our daily activities, such as information retrieval or task organization. 20 years later, portable digital devices, such as smartwatches, notebooks, tablets or smartphones, are widely available and easily accessible. We spend more and more time on, e.g., social media, video calls, internet browsing, Netflix watching etc. A digital world, risen with the internet, has become more prominent and pervasive in our lives. The vision that we carry ubiquitous technology around all the time and that it is part of our existence has become reality.

Ubiquitous technology draws our attention towards itself and consequently to the whole digital world of information, social networks, etc. this world provides. Like in daydreaming (i.e., being in a mental world), people easily get absorbed by the digital world and forget about their surroundings, i.e., the real, physical world. For example, everyone fantasizes about their own wishes from time to time and might be disappointed when being ripped out of the dreams, realizing that it never existed in the real world. Similarly, we are all familiar with the situation of having a coffee-table conversation interrupted by a ringing phone. Be it a message or a call both can rip us out of a flowing conversation and create an awkward moment for all parties involved. Worse than awkward, typing a message while driving a car creates a conflict causing a dangerous moment. Waterworth et al. [16, p.6] point out that it is "not only a conflict between presence here and presence there, but often also a conflict between self and other, presence and absence".

In computer science, there is a plethora of definitions and conceptualizations of the concept of presence (see, e.g., $[6,7,13]$ for a review). Witmer et al. [17, pg.1] consider presence to be "a psychological state of 'being there' mediated by an environment that engages our senses, captures our attention, and fosters our active involvement". Although the environment can be "real, virtual, symbolic, or some combination thereof" [17, pg.1], in HCI, the environment is commonly considered a virtual environment. Presence is most famously known and researched in the field of virtual reality (VR), as the feeling of being in the technology mediated environment (sometimes also referred to as "telepresence" [11]). Research on presence in VR focuses on maximizing presence in the virtual (digital) world while minimizing the awareness of the real world as much as possible. Only recently, VR researchers began to 
acknowledge the need for and benefits of balancing presence in multiple worlds rather than being only in the virtual reality [3] At the same time, voices, which raise concern over everyday ubiquitous devices taking up to much space of our lives, are getting louder - calling for restraining digital device usage (i.e., digital detox) $[14,15]$. In the context of this paper, a virtual environment accessed by everyday ubiquitous devices is referred to as digital world. In contrast, a virtual environment accessed a by an immersive VR device, e.g. head mounted display, is referred to as virtual reality.

Instead of going to the extremes of moving the presence fully to a digital world or completely cutting off everyday digital devices, we believe there is a middle ground worth exploring. As the examples above illustrate, presence is not only a topic relevant for VR and head-mounted displays anymore, but also concerns ubiquitous technology. At the same time, those examples and the digital detox trend show that people have troubles with being present in the real world and managing their dives into the digital one. HCI research has already identified the need to support users in managing their technology use with, for example, time-tracking apps [4] and paradigms for less pervasive smartphone interactions [12]. Although only indirectly, they also help managing one's presence in the real world, as the access to the digital world is limited. Completely cutting of the digital world may be a solution for the moment but not for the long-term. Today, it is nearly impossible to live a modern life with all its benefits without encountering and using technology at work as well as at home. In this paper, we take a step back and make initial explorations of presence in the era of everyday ubiquitous technology. We are particularly interested into what people assume their feeling of presence is and how ubiquitous technology influences this perceived feeling, as managing our presence across the real and digital world is important and "necessary for effective action in the world" [16, p.6].

With the goal of understanding the dynamics of presence in everyday life, under the existence and usage of everyday ubiquitous devices, we first conducted a focus group with $N=6$ presence experts. We gathered insights into their conceptualizations of presence in the real and digital worlds. Following, we performed an online survey with $N=36$ non-experts. We similarly gathered insights into their conceptualizations of presence across the real and digital world, but were also interested into how one world may affect the other. This particularly includes individual experiences and situations of moving between the worlds as well as the motivations and effects of such. In this exploratory work, we discuss novel research questions and theories based on our preliminary results.

\section{STUDY DESIGN}

We took a twofold approach to explore users' conceptualization of presence and its relation to technology: Firstly, we completed a focus group $(\mathrm{N}=6)$ to gather insights into experts' conceptualizations of presence and find a way of how to communicate the idea best to non-experts. Secondly, we completed an online survey $(\mathrm{N}=36)$ to explore whether non-experts have a (clear) concept of presence in mind and how they experience and describe their presence in everyday situations including ubiquitous technology.
Table 1: Overview of our focus group participants.

$\begin{array}{lcc}\text { Background } & \text { Gender } & \text { Age } \\ \text { Psychologist (Doctoral Candidate) } & \text { M } & 29 \\ \text { Psychologist (Children Social Worker) } & \text { M } & 28 \\ \text { VR Researcher (Doctoral Candidate) } & \text { M } & 34 \\ \text { HCI Professor \& Experienced Meditator } & \text { M } & 60 \\ \text { AI Ethics Researcher \& Experienced Meditator } & \text { F } & 39 \\ \text { HCI Student \& Meditator } & \text { F } & 25\end{array}$

\subsection{Study 1: Focus Group}

The aim of the focus group was to (1) understand experts' notion of presence and their formulation of it as well as to (2) explore whether and when they have a feeling of being in different worlds and the role of technology in such. We aimed to thereby gather first insights into peoples' concepts of presence and to help the design of subsequent research steps.

2.1.1 Method. Before the beginning, we communicated that the session would be recorded and obtained participants' consent to do so. In a short introductory round we carefully explained the aim of the focus group with particular attention on not to describe the meaning of presence and bias their own understanding. We started the first part by requesting participants to silently reflect on and write down their understanding of presence and terms they relate to it. The reflection phase ensured a deliberate contemplation and recall to form their conception of presence.

We then asked each participant to read out the own notes to the group. Only after everyone shared, we encouraged a discussion among the participants and asked them to identify a set of factors that influence their feeling of being present. As we expected participants to have different views on its meaning, we aimed to, thereby, establish a mutual understanding or an awareness of what it means to others, i.e., its various meanings, facets and factors. The focus group lasted for overall one hour.

2.1.2 Participants. Participants were recruited through our extended network and via direct email. Recruitment was based on people's experience with presence in varying environments, which we derived from a literature review, e.g. mediation or VR research. They joined on a voluntary basis and were compensated with breakfast. Table 1 provides an overview of the participants.

\subsection{Study 2: Online Survey}

The aim of the online survey $(N=36)$ was to investigate (1) nonexperts' understanding of the term presence and (2) consolidate everyday situations in which technology influenced their presence in the real or digital world.

2.2.1 Method. Within this paper, we report on a subset of data from an online survey, including seven questions that required Likert-Scale responses (see figure 2) and two questions that asked for written responses: 
Q1 Please explain what you understand under presence and being present. Your understanding may very well differ from what we explained above.

Q2 Please try to recall and then shortly describe a situation from the last days in which technology has influenced your feeling of presence either negatively or positively.

As we were unsure whether people have formed a concept of presence and whether presence plays a role in people's everyday lives, we provided a brief introduction to the common understanding of presence in the beginning of the survey, based on our insights from the focus group. Yet, we also made clear that there is ample room to re-define it by the participants themselves. See Fig 1 for the complete text we provided in the survey's introduction.

2.2.2 Participants. We recruited participants via university mailing lists and social media networks. 38 participants completed the survey, with two participants being excluded from the final set as they provided no reasonable answers across all open-ended questions. This resulted in a final set of $N=36(\mathrm{~F}=24$, Other=1) participants. They were on average 28 years young $(\min =19, \max =69$, $S T D E V=10,76)$, with a diverse educational and vocational background.

\section{RESULTS}

In this section we present the combined results of the focus group [FG] and the online survey [OS]. Data from the focus group was analyzed through a thematic analysis. Qualitative data from the online survey was analyzed by using grounded theory with an inductive approach. Likert-scale responses from the online survey are presented in figure 2 .

In alignment with our research goal, results are organized in (a) participants understanding of presence, and (b) effects of technology on presence.

\subsection{Understanding of Presence [FG \& OS]}

As anticipated, the understanding of presence differed based on experts' professional background (e.g., HCI, psychology etc.), as well as their experience with meditation practices. Experts' notions of presence generally center around the awareness of the current situation and how the self relates to the environment, for example: (a) dealing with the here and now [5], (b) interacting with the world around, (c) social presence [8]; i.e., the presence of one self and how one relates to other people in their surroundings (e.g., "annoying", "actively listening"), (d) telepresence [10]; i.e., taking part in a remote communication or activity, and (e) spatial $[2,18]$ and social presence (in VR) [2].

The discussion of the different notions was influenced by the unusual view of one participant with extensive meditation background and dwelt on the interpretation of presence in the eastern and western culture, i.e., non-dualism vs. dualism of the self and its environment [19].

Regarding the online survey, one participant expressed agreement with our explanation, whereas one participant did not understand it. Although we cannot be certain to what extent our introduction influenced their definition, all participant provided their own definition, suggesting a low bias through the introduction. Our participants differentiated between the notion of presence and being present. Whereas for some of the participants, presence meant a physical, embodied presence (i.e., as in being somewhere physically, or simply existing), being present was mostly understood as the joint presence of body and mind (P135: “...being there with mind and heart."). In that sense, presence was contemplated as (focused) attention on something or the awareness of the self and surroundings (P83: "I am present in a certain situation when I concentrate on what is happening and I feel like I am a part of the world around me, not being distracted or detached from the events."), often in that particular moment (P36: "Being present means being totally focused on what happens right now - without any thoughts concerning the past, the future or your fantasies (e.g., daydreams)". Some participants understood presence as their ability to absorb information from their surroundings and, consequently, their readiness to promptly (re)act to information (P46: "I'd say being present means taking in the content presented by the respective "world". I can sit in my living room, but only take in content of the digital world, but I can also sit in front of computer with a video on and not take that in because I'm daydreaming. That would probably be one of the scenarios in which I'm technically participating in all three worlds, but only take content in in one, which is the mental one."). Many participants strongly tied a social dimension to presence, as in, how they are perceived by others (e.g., as P87 stated: "Presence to me means being physically present. E.g., my presence can be perceived by others by them looking, touching, listening etc."), as well as what their role in the society is, as P82 contemplated: "Being present revolves around being aware of your surroundings. This includes being aware of your role in the community, or aware that there are others around you also sharing thoughts and feelings that you do. Presence is thinking beyond yourself."

\subsection{Exploring the Interplay Between Worlds: Effects of Ubiquitous Technology on Presence [OS]}

We set out to explore scenarios in which users manage their presence, with technology involved. Two researchers separately coded the data from the online survey and later on discussed their findings and formed themes.

Control forms the overarching theme, whereby we differentiate between whether the user has control over their presence or their presence is controlled by external entities. Depending on the amount of control, taking away the sense of presence in the real world can be perceived as something positive or negative. When in control, technology offers a deliberate escape from the real world, with the goal of being somewhere else, being someone else or being somewhere else with someone else. When not in control, this escape corresponds to an undesired loss of presence. Similarly, users utilize technology (or not) for the purpose of controlled competence or time gain. Yet, when out of control, users associate the use of technology with an overload. Below we provide an overview of our results with illustrative quotes for internal vs. external control over presence.

3.2.1 "Being Somewhere Else": Escaping from vs. Losing Awareness of Surroundings. Using technology can induce the feeling of being somewhere else than participants' physical location. When in users' 


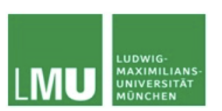

\title{
Presence: Being present in the real, mental and digital world.
}

Introduction

\begin{abstract}
We are very used to always have the real world around us and our mental world inside us. Nowadays, technology is an integral part of our lives and people spend increasingly more time on social media, for example, being in calls, surfing the net, watching Netflix etc. Through technology we access a "digital world" and this world is becoming more and more pervasive and important to us - with smartphones most people carry the "digital world" in their pockets all the time.

The borders between these three worlds are blurry. It is very individual to what extent we dive into each of these three worlds and some of us may be in one world only at a time while others may balance themselves across all three. With this survey, we want to better understand how people navigate between these three worlds and what strategies they use to do so. In that context, we want to distinguish these three worlds as described below. This may seem abstract at first but it will get more concrete when we move on please bear with us.

- With "real world" we refer to the physical world around you. This includes everything that you can see, hear and touch (and does not require electricity for that). For example, your smartphone is part of the real world because its a physical object that you can hold it in your hands but the content you see when you turn it on, belongs to the digital world.

- With "digital world" we mean anything that is accessible to you through technology. This world includes, for example, the internet, online social media like Facebook, TV shows, etc.

- The term "mental world" refers to the thoughts that you have in your mind. This includes, for example, when you brainstorm about something, daydreaming, but also the focus on breath in meditation.

We use the terms "presence" and "being present" to capture the feeling of BEING in these worlds. For example, as you fill the survey, do you feel more present in what is around you (real world) or in your computer (digital world)? With this survey we also want to find out what being present means to people and what makes them feel present. Remember, this is very individual and the borders between the worlds are blurry. Hence, there are no right or wrong answers.

In the survey, we will ask you about your personal understanding and perception of presence in the different worlds and how you manage them. We, furthermore, would like you to imagine yourself in a given scenario and explain your presence in the different worlds and the strategies you use to navigate between them.
\end{abstract}

Figure 1: The on-boarding text on the notion of presence across three worlds, that we provided to our non-expert group in the online survey.

control, it can present a method of escape from an uncomfortable setting, as P108 writes: "I remember being at a party (I didn't want to go to) and feeling extremely bored. I deliberately took my phone and started working on my emails and stuff to feel "somewhere else"."

Depending on the amount of control over presence in the real and digital world, it can both be a pleasant or disturbing experience, as P87 states: "When I was focusing on a Youtube series too much, I felt my awareness of my physical surroundings has decreased to a minimum. Only after a certain amount of time I felt that I lost touch with this real world around me, which sometimes is nice and sometimes shames me."

Being around technology led some participants to be not present in the real world due to external or internal triggers. P113's reported a situation of being pushed out of the real world by an external entity (i.e., technology): "I was at a party and as soon as I got a message from someone at my phone, I was not present in the real world anymore." On the other hand, P127 was herself drawn to technology: "I was watching a movie with my boyfriend and I felt the urge to check my Whatsapp messages after an hour, but that made me feel less present in the moment."

3.2.2 "Being Someone Else": Escaping vs. Losing Myself. Technology can induce the feeling of being someone else. In case of users' control, it presents a pleasant escape, a fantasy. Yet, the standards set in the digital world can be tough to achieve, leading to a hard awakening from the fantasy, as P36 illustrated: "[...] many YouTube-Videos or homepages on the internet lead to me fantasizing about ('re-')living the same or similar situations as depicted in the video or the website. This on the one hand can be positive, because it can make me feel better for some time. On the other hand it can be very negative, because it leads to me wasting this exact moment and not living my own potential to the fullest. Furthermore, sometimes I "wake up" from my fantasy and realize, that I can never be like the persons shown in the video or will never do, what they do. This on the other hand makes me feel bad about me and can lead to inferiority complexes."

P36 statement pictures a recurring pattern of trying to identify themselves with characters from the digital world. Total capture in the digital world requires re-orienting oneself once back in the real world: "While watching a movie I was completely captured in the shown world. I was pretty surprised to find myself in the 'real world' after it ended. Because the characters changed I was not able to identify myself with one, which led to a state without a physical awareness or even an awareness for me as an individual. So my feeling for being present was not there. Nonetheless, I was present in that world."

In cases one's presence is reduced already, technology can have a rather spiraling effect, as $\mathrm{P} 39$ said: "However in the past few days, due to tiredness I don't feel present anymore. And every time I spend time on my phone, this blurriness in my existence is deepened." 


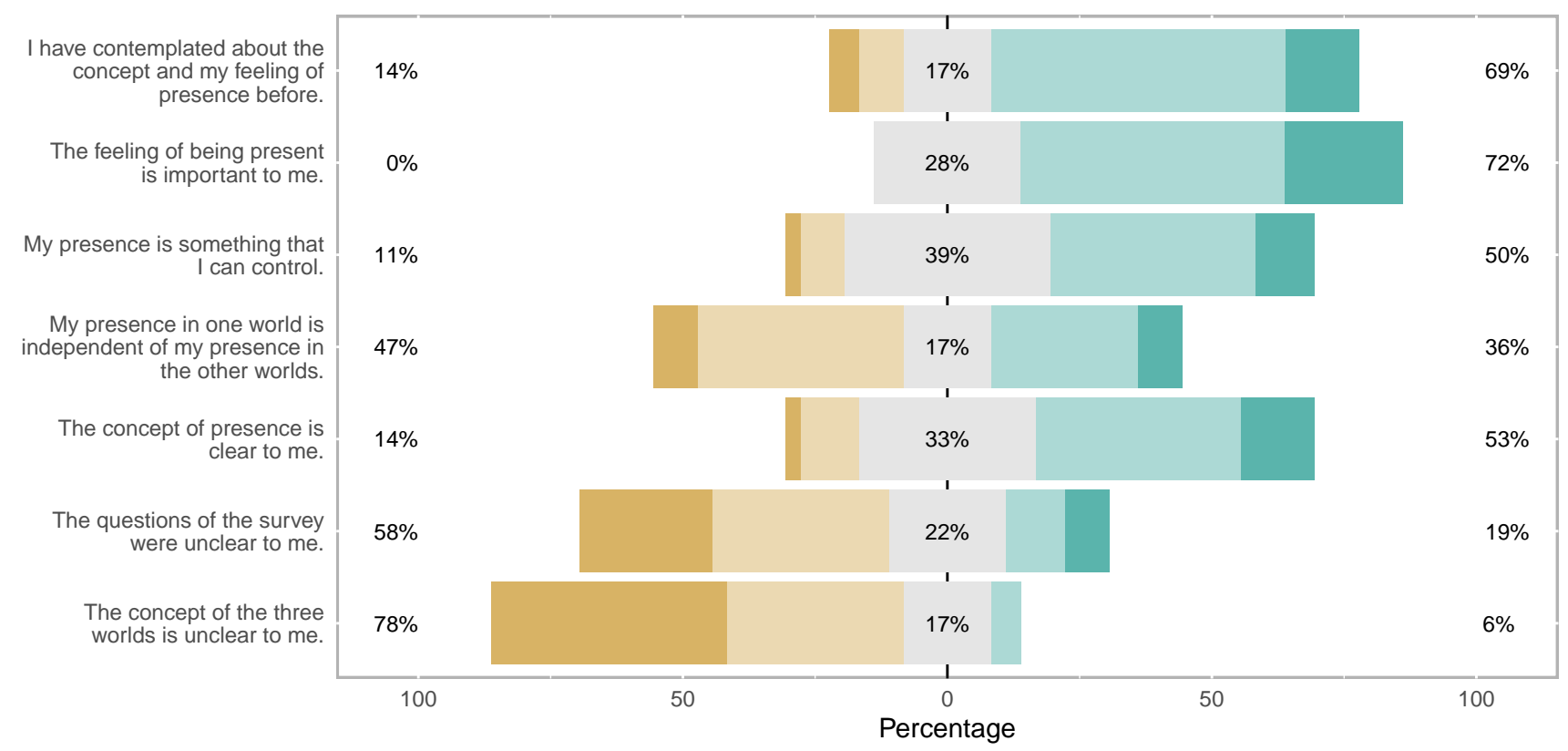

\begin{tabular}{l|l|l|l|l|l|l} 
Response & strongly disagree & disagree & neither agree nor disagree & agree & strongly agree
\end{tabular}

Figure 2: The distribution of participants' $(N=36)$ responses to the 7 Likert-scale questions from our online survey. The responses show that more than $2 / 3$ of our participants have contemplated about their presence before and consider presence to be of importance to them. Half of the participants are convinced that they can control their presence, although just as many of them think that presence in one world is not independent of their presence in the other worlds.

3.2.3 "Being Somewhere Else with Someone Else": Feeling Connected vs. Trapped. The easy accessibility of everyday technology enables users to be with people who are physically distant and even in other time zones. Technology, thus, moves their presence through time and space, inducing a feeling of presence with a particular person and at another place. For example, P46 described: "it was my brother's birthday a couple of days ago and I could congratulate him at midnight (his time zone) even though I live in another country than the rest of my family. He saw it minutes later and responded, which made me feel connected to him."

On the other hand, some participants feel trapped in the digital world, never fully able to break free, as P82 illustrated: "Technology enables us to always be available for communication and contact, no matter what part of the world you are on. I have family and friends in different countries, and with technology, getting away never really seems like an option, since it is nearly always possible to contact me."

3.2.4 "Doing More": Gain vs. Loss of Competence. Some participants mentioned an increase or decrease of competence or performance due to technology. P147 noticed a performance improvement in their task execution in the real world, while being distracted by the digital world: "I noticed that during sport time, [I was biking] more quickly when I was playing on my phone."

However, P79 remarked that inability to keep up with advances in technology can make one feel isolated or not present with society:
"Since I don't know much about new Technologies and have a hard time learning how to use them it distances myself and the others sometimes. For me in some old fashioned way the things many times still have to be touched etc... it is a lot easier for me when I have the things in my hand than just to have them on screen, I feel distanced from society because most people in my age don't have problems with these technologies at least those with an academic background."

3.2.5 Loss of Time. There is a common belief among our participants that time flies when they are in the digital world. For example, P63 explained: "I was on a trip home, looked up something on my phone and was shocked at how fast time passed in the meantime." In the same vein, P106 mentioned: "I was hiking lately and there was no WiFi available... I felt much more present and time felt extended." Accordingly, users perceive to have more time at disposal when their presence is exclusively bound to the real world, ultimately leading to a stronger feeling of presence.

\section{DISCUSSION \& FUTURE WORK}

We conducted two preliminary studies, an expert focus group $(N=6)$ and a non-expert online survey $(N=36)$, to explore (1) people's understanding of presence, and (2) if and how they manage their presence when a digital world is continually at their fingertips.

Our results show that users' understanding of presence - across both expert and non-expert groups - is generally in alignment 
with prior work, meaning that researchers and users do have a common understanding. Our participants identified main themes from prior work $[13,17]$ that affect presence, such as the ability to interact with the world around, social aspects, communication with others and the environment itself. The results, furthermore, showed that people are aware of their presence, but not always in control of it - leading to dissonances in the allocation of their presence across the worlds and undesired feelings. Starting from that point on, we revealed three major themes that we discuss in form of novel research questions and theories for future work below.

What does "being in the digital world" even mean? Only few participants described having a sense of presence in a world other than the real one. This raises the question whether calling it presence when one feels to 'be in the digital world' is just uncommon to them or whether it really is a different feeling for them compared to being present in the real world. Yet, nearly all descriptions clearly show them diving into another world and forgetting about the real one around to some extent. Our results also show that this change can take time, indicating that it can be a slow shift rather than an immediate switch: Like a spectrum with the real world on one end and the digital one on the other and presence as the shifter. Although this spectrum may overlap with Milgram's Reality-Virtuality continuum [9], its difference lies in the fact that presence can be achieved independent of the immersion that the underlying technology provides.

Finding the right balance. Most of the current research either aims to maximize presence in the digital world (i.e., VR) or to exclude the same completely and focus presence on the real world (digital detox). In contrast to aiming for those extreme ends, we showed that there is a gray space in between. We, furthermore, argue that it is worth exploring this scale, as we can neither exist only in the real nor in the digital world nowadays - we need to find a balance. Yet, it seems that balancing presence along that spectrum is as walking along a thin wire. Few of our participants' reports illustrated scenarios in which a person truly managed to balance the digital and real world at a time. On the contrary, the scenarios include lots of transitions and conflicts between worlds, i.e., deliberate switches as well as undesired moves back and forth. This makes us question whether people can divide a capacity of presence and share it across the worlds or whether people have only one atomic presence which they shift from one world to another? And in what way can we support people in both cases with technology? We believe that understanding this difference changes the technology design for supporting the transitions between the worlds. Our results indicate that people have established strategies to manage their presence across the worlds. Consequently, one may suggest that ubiquitous technology could support people in that. Some strategies and features already exist, such as tracking the time spent on smartphones [4]. Do the strategies differ depending on technology or task and are they transferable (e.g., transitioning back to the real world from a TV vs. PC experience)? How can technology support these strategies?
What knowledge can we transfer from VR? There appear countless other questions when (1) contemplating about the concept of presence out of the box of VR and extending it to ubiquitous technology as well as when (2) regarding it as a multi-directional construct including three worlds (real, digital and mental), rather than only aiming to increase presence in one and excluding the others. VR research does provide some landmarks [3] and mobile app researchers have acknowledged the gap in design recommendations [12] to manage presence. However, we argue for a more complete map to the landscape of presence as well as signposts that navigate designers of everyday ubiquitous technologies to help users move smoothly through and/or across the worlds, i.e., balance their presence across the worlds.

\section{CONCLUSION}

Ubiquitous everyday devices, such as smartphones or notebooks, enable easy and fast access to a digital world - in addition to the real world surrounding us and the mental world within us. This makes our presence increasingly shift between worlds - with one more world than we naturally have, causing conflicts and uncomfortable feelings but also opening a whole world, in which we can explore, imagine and grow in manifold ways. Within this work, we made an initial exploration of user's understanding of presence and the different worlds as well as of their experiences with moves and management of such. We, therefore, conducted a focus group $(N=6)$ and subsequent online survey $(N=36)$. Our findings demonstrate lots of (un)controlled transitions between the real and the digital world, calling for an exploration of strategies to balance those transitions in everyday life.

In the next steps of our research, we will more deeply analyze peoples' transitions between the worlds as well as their strategies to switch or remain present in one world. Furthermore, we will investigate the factors that influence their control over the transitions as well as those factors that make a strategy successful.

\section{REFERENCES}

[1] Gregory D Abowd and Elizabeth D Mynatt. 2000. Charting past, present, and future research in ubiquitous computing. ACM Transactions on Computer-Human Interaction (TOCHI) 7, 1 (2000), 29-58.

[2] Frank Biocca, Chad Harms, and Judee K Burgoon. 2003. Toward a more robust theory and measure of social presence: Review and suggested criteria. Presence: Teleoperators \& virtual environments 12,5 (2003), 456-480.

[3] Ceenu George, An Ngo Tien, and Heinrich Hussmann. 2020. Seamless, Bidirectional Transitions along the Reality-Virtuality Continuum: A Conceptualization and Prototype Exploration. In 2020 IEEE International Symposium on Mixed and Augmented Reality (ISMAR). IEEE, 412-424.

[4] Alexis Hiniker, Sungsoo Hong, Tadayoshi Kohno, and Julie A Kientz. 2016. MyTime: designing and evaluating an intervention for smartphone non-use. In Proceedings of the 2016 CHI Conference on Human Factors in Computing Systems. 4746-4757.

[5] Jon Kabat-Zinn. 2015. Mindfulness. Mindfulness 6, 6 (2015), 1481-1483.

[6] Kwan Min Lee. 2004. Presence, explicated. Communication theory 14, 1 (2004), $27-50$.

[7] Matthew Lombard and Matthew T Jones. 2015. Defining presence. In Immersed in Media. Springer, 13-34.

[8] Patrick R Lowenthal. 2010. Social presence. In Social computing: Concepts, methodologies, tools, and applications. IGI Global, 129-136.

[9] Paul Milgram, Haruo Takemura, Akira Utsumi, and Fumio Kishino. 1995. Augmented reality: A class of displays on the reality-virtuality continuum. In Telemanipulator and telepresence technologies, Vol. 2351. International Society for Optics and Photonics, 282-292.

[10] Marvin Minsky. 1980. Telepresence. (1980).

[11] International Society on Presence Research. 2002. About presence. https://ispr. info/about-presence- $2 /$. 
[12] Simon Robinson, Gary Marsden, and Matt Jones. 2014. There's not an app for that: Mobile user experience design for life. Morgan Kaufmann.

[13] Richard Skarbez, Frederick P Brooks, Jr, and Mary C Whitton. 2017. A survey of presence and related concepts. ACM Computing Surveys (CSUR) 50, 6 (2017), $1-39$.

[14] Trine Syvertsen and Gunn Enli. 2020. Digital detox: Media resistance and the promise of authenticity. Convergence 26, 5-6 (2020), 1269-1283.

[15] Naciye Guliz Ugur and Tugba Koc. 2015. Time for digital detox: Misuse of mobile technology and phubbing. Procedia-Social and Behavioral Sciences 195 (2015), 1022-1031.
[16] John Waterworth and Giuseppe Riva. 2014. Feeling present in the physical world and in computer-mediated environments. Springer.

[17] Bob G Witmer, Christian J Jerome, and Michael J Singer. 2005. The factor structure of the presence questionnaire. Presence: Teleoperators \& Virtual Environments 14, 3 (2005), 298-312.

[18] Bob G Witmer and Michael J Singer. 1998. Measuring presence in virtual environments: A presence questionnaire. Presence 7, 3 (1998), 225-240.

[19] Michael E Zimmerman. 1993. Heidegger, Buddhism, and deep ecology. The Cambridge Companion to Heidegger (1993), 240-69. 